and promising new technique: those working on peptide synthesis will await the outcome of this rigorous evaluation eagerly.

The meeting concluded with two communications dealing with studies of structure-activity relationships using synthetic peptides. R. L. Strong and H. D. Law of Liverpool Regional College of Technology and M. J. Crumpton of the National Institute for Medical Research were concerned with the immunological activity of peptides related to one of the antigenic determinants of sperm whale myoglobin, and $\mathbf{H}$. $R$. Perkins and M. Nieto of the National Institute for Medical Research described a study of the specificity of the interaction of peptides related to bacterial coll walls with the antibiotic vancomycin. Unfortunately this interaction, which is presumably involved in the action of the antibiotic, cannot yet be interpreted in molecular terms because the structure of vancomycin is still obscure.

\section{PROTEIN SYNTHESIS}

\section{Beginning and Middle}

from our Cell Biology Correspondent

THE structural features which distinguish those species of methionine $t$ RNA which can be formylated, and which act as initiators for protein synthesis, from other methionine $t$ RNAs must be very subtle indeed. It had been hoped that a simple comparison of the complete nucleotide sequences of $E$. coli $t \mathrm{RNA}^{\text {met }}$ and $t \mathrm{RNA}_{\mathrm{F}}^{\mathrm{m}}$ would reveal the distinguishing features which allow the latter $t$ RNA to initiate protein synthesis, but these hopes were dashed last year when Dube, Marcker, Clark and Cory (Nature, 218, 232; 1968) reported the sequence of the $E$. coli initiator, $t \mathrm{RNA}_{\mathrm{F}}^{\text {met }}$. There were only three obvious differences between this molecule and $t$ RNA $^{\text {m.t. }}$. First, except in yeast valine $t$ RNA, of the $t$ RNAs analysed so far, including $t \mathrm{RNA}^{\mathrm{mct}}$, the base following the anticodon is either a modified form of adenosine or a rare base such as methyl inosine, whereas in the initiator $t \mathrm{RNA}_{\mathrm{F}}^{\text {met }}$ the base following the anticodon is an unmodified adenosine. Perhaps this unmodified base accounts for the unique ambiguity of the $t \mathrm{RNA}_{\mathrm{F}}^{\mathrm{m} t \mathrm{t}}$ which recognizes two codons $\mathrm{AUG}$ and GUG. The second difference is in a part of the molecule
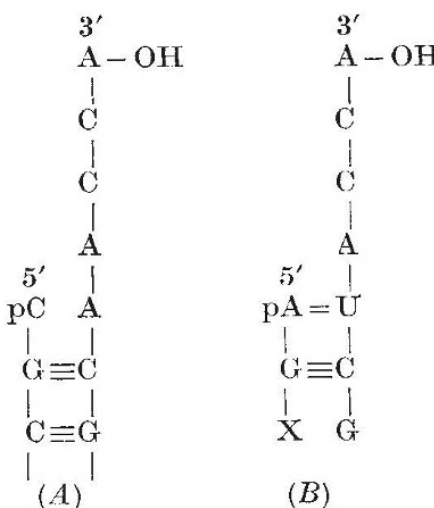

$$
\text { (A) }
$$

Fig. 1. The terminal nucleotide sequences of $(A) E$. coli $t R A_{F}^{\text {met }}$ and $(B)$ yeast $t R N A_{F}^{\text {met }}$. The $E$. coli $t R N A$ is unique in that base pairing involves the sixth but not the fifth base from the $3^{\prime}$ end. All other tRNAs analysed so far are like the yeast $t R^{\prime} A_{F}^{\text {met }}$ with the fifth base from the $3^{\prime}$ end paired with the $5^{\prime}$ terminal base. which contains a pseudo-uracil residue, and its significance is obscure. The third difference is in the base pairing of the $3^{\prime}$ and $5^{\prime}$ ends of the molecule; at the time it was suggested that this might provide a unique recognition site for the transformylase enzyme which formylates methione residues attached to $t \mathrm{RNA}_{\mathrm{F}}^{\text {met }}$ but not those attached to $t$ RNA ${ }^{\text {uut }}$.

This attractive suggestion has now been ruled out by a partial analysis, by RajBhandary and Ghosh (J. Biol. Chem., 244, 1104; 1969), of the nucleotide sequence of a yeast methionine $t$ RNA, which can be formylated by the $E$. coli transformylase enzyme and initiate protein synthesis in a cell freo system. In yeast there are two species of methionine $t$ RNA but only one of these can be formylated. The complete nucleotide sequence of this species has yet to be determined and it is not yet known whether the base immediately following the anticodon is unmodified as in $E$. coli $t$ RNA $_{\mathrm{F}}^{\text {met }}$. RajBhandary and Ghosh have, however, determined the sequences of the $3^{\prime}$ and $5^{\prime}$ ends of the yeasts $t \mathrm{RNA}_{F}^{\text {met. }}$. Apparently these two sequences are like all the other $t$ RNAs except the $E$. coli initiator in that base pairing involves the fifth base from the $3^{\prime}$ end and the $5^{\prime}$ terminal base. The figure shows the difference between the yeast and $E$. coli $t \mathrm{RNA}_{\mathrm{F}}^{\text {met }}$ sequences at the termini.

The significance of this, of course, is that the unusual base pairing of the termini of the $E$. coli $t \mathrm{RNA}_{\mathrm{F}}^{\text {met }}$ cannot any longer be regarded as a recognition site for the transformylase enzyme, because this enzyme recognizes and formylates $t$ RNAs with both the sequences shown in the figure. It seems that the unique structural features of initiator $t$ RNAs will not be recognized until the three-dimensional structure of the molecule has been determined by X-ray crystallography.

\section{PROTEIN STRUCTURE}

\section{More Disulphide Exchange}

\section{from our Molecular Biology Correspondent}

LAST week I commented on the interesting results from Foster's laboratory, which indicate that the native conformation of bovine serum albumin is not unique, in the sense that several disulphide pairings scem to correspond to stable structures, which can coexist in equilibrium with one another. This situation was recognized as a violation-or at least a modificationof the law that the sequence of a polypeptide chain defines a unique conformation, a rule which has so far always seemed to hold good in enzymes.

A nice demonstration of this principle, which was first clearly enunciated by Anfinsen, comes now from Kato and Anfinsen (J. Biol. Chem., 244, 1004; 1969). In previous work at the same laboratory, a protein was prepared from liver microsomes which catalyses the refolding of denatured proteins. It does this by facilitating disulphide interchange, in which it must itself participate as an intermediate; the renaturing protein, which is searching for the conformation of minimum energy, is then less liable to trapping in metastable states produced by incorrect disulphide pairings, which otherwise impede renaturation. The system which Kato and Anfinsen have examined is ribonuclease-s', in which, it will be recalled, the chain is broken twenty residues from the end. The icosapeptide (S-peptide) can be romoved, leaving an inactive $S$-protein. but 